\title{
Magnetic Reconnection on Jet-Accretion disk Systems
}

\author{
Elisabete M. de Gouveia Dal Pino* \\ Instituto de Astronomia, Geofísica e Ciencias Atmosfericas - Universidade de São Paulo \\ (IAG/USP), São Paulo, Brazil \\ E-mail: dalpino@iag.usp.br
}

Maria Victoria del Valle

Instituto Argentino de Radioastronomäśa (IAR), Argentina

\section{Luis Kadowaki}

IAG - Universidade de São Paulo, Brazil

\section{Behoruz Khiali \\ CERN, Switzerland}

\section{Grzergorz Kowal}

EACH - Universidade de São Paulo, and UniSul, Brazil

\section{Yosuke Mizuno}

Institute for Theoretical Physics, Goethe University, Germany

\section{Chandra B. Singh}

IAG - Universidade de São Paulo, Brazil

Fast Magnetic Reconnection is currently regarded as an important process also beyond the solar system, specially in magnetically dominated regions of galactic and extragalactic sources like the surrounds of black holes and relativistic jets. In this lecture we discuss briefly the theory of fast magnetic reconnection, specially when driven by turbulence which is very frequent in Astrophysical flows, and its implications for relativistic particle acceleration. Then we discuss these processes in the context of the sources above, showing recent analytical and multidimensional numerical MHD studies that indicate that fast reconnection can be a powerful process to accelerate particles to relativistic velocities, produce the associated high energy non-thermal emission, and account for efficient conversion of magnetic into kinetic energy in these flows.

Frontier Research in Astrophysics - II

23-28 May 2016

Mondello (Palermo), Italy

\footnotetext{
*Speaker.
} 


\section{Introduction}

Black Holes (BH) and the associated accretion and relativistic jet phenomena are ubiquitious in Astrophysics. There are evidences for the presence of stellar-mass BHs in galactic binary systems, usually named GBHs (or BHBs, or microquasars). Also, supermassive BHs (with $\sim 10^{6}$ to $10^{10}$ solar masses) are believed to occur in the nuclei of all classes of active galaxies (or AGNs, from the high luminous blazars to the less luminous radio or seyfert galaxies). Besides, it is also argued that BHs may be the ultimately engines of gamma-ray-burts (GRBs).

Multi-wavelength observations indicate that the collimated relativistic jets produced near the central $\mathrm{BH}$ can be accelerated to large Lorentz factors, up to several orders of magnitude in length scales. Their formation is still a matter of debate, but the usually most accepted models rely on magnetic processes, like magneto-centrifugal acceleration by helical magnetic fields arising from the accretion disk [1]. Or otherwise they can be powered by the BH spin transferred to the surrounding magnetic flow [2]. Thus in any case the prediction is that the jets should be born as magnetically or Poynting flux dominated flows. The fact that at observable distances from the source (starting around $1000 R_{S}$ or less, where $R_{S}$ is the Schwartzschield radius) these jets become kinetically dominated, indicate that they must somehow suffer an efficient conversion (or dissipation) of magnetic into kinetic energy. We argue below that magnetic reconnection may be a powerful mechanism operating in these jets to allow for this conversion.

Another problem that is currently challenging the researchers regards the origin of the very high energy (VHE) emission of these sources. For instance, until recently only AGNs with highly beamed jets pointing to the line of sight, namely blazars, were detected by gamma-ray telescopes. More than a chance coincidence, these detections are consistent with the conventional scenario that attributes the VHE emission of these sources to particle acceleration along the jet being strongly Doppler boosted and producing apparently very high fluxes. Recently, however, a few non-blazar sources which belong to the branch of low luminosity AGNs (or simply LLAGNs) for having bolometric luminosities of only a few times the Eddington luminosity, have been also detected at $\mathrm{TeV}$ energies by ground based gamma-ray observatories (e.g. [3] and references therein). Among these sources, the radio galaxies M87, Centaurus A (or Cen A), Persus A (or Per A), and IC 310 are the most striking examples. The angular resolution and sensitivity of these detectors are still so poor that it is hard to establish if it comes from the jet or from the core. These VHE detections were surprising because, besides being highly underluminous, the viewing angle of the jets of these sources is of several degrees, therefore allowing for only moderate Doppler boosting. These characteristics make it hard to explain the VHE of these sources adopting the same standard scenario of blazars. Furthermore, observations of short time scale variability in the gamma-ray emission of IC 310, M87 and Per A (e.g., [4, 5, 6, 7] and references therein) indicate that it is produced in a very compact region that could be perhaps the core. In the case of Cen A, though there is no evidence of significant variability at VHE, it has been also argued that the $\mathrm{TeV}$ data of this source would be more compatible with a point source near the core [8].

Though a number of works have attempted to explain these observations as produced in the jets of these sources (e.g. $[9,10,11])$, the evidences above led to the search for alternative particle acceleration scenarios involving the production of the VHE in the surrounds of the BH, in the core region (see e.g. $[12,13]$ and references therein). If such environments are magnetically dominated 
as generally supposed, then fast magnetic reconnection may be unavoidable [16, 17].

Researchers have been facing similar difficulties also with the interpretation of the VHE emission of GBHs and their jets, particularly with the sources Cygnus X1 and X3 for which recent detections indicate upper limits in the $\mathrm{TeV}$ range too $[12,18,19]$.

Even in the cases where the emission is most probably produced along the relativistic jets, as in blazars, the conventional mechanism to accelerate the particles based on diffusive shock acceleration is facing severe constraints imposed by current VHE observations with very high variability, of the order of minutes in the $\mathrm{TeV}$ range. This implies extremely compact acceleration/ emission regions $\left(<R_{S} / c\right)$ and besides, it requires bulk Lorentz factors larger than the typical values expected for such sources (around $\gamma \simeq 5-50$ ) in order to avoid electron-positron pair creation. To circumvent these problems, [20] proposed a reconnection model involving misaligned mini-jets inside the jet. Fast reconnection has been also invoked in [21] to explain the transition from magnetically to kinetically dominated flow and the prompt gamma-ray emission in GRBs.

In this lecture, we discuss the role of fast magnetic reconnection in accretion disk/jet systems around $\mathrm{BHs}$ and show that this can be a powerful process to accelerate relativistic particles, produce the associated non-thermal emission and particularly the VHE one, and account for an efficient conversion of magnetic into kinetic energy.

\section{Fast Magnetic Reconnection and Particle Acceleration}

Fast magnetic reconnection occurs when two magnetic fluxes of opposite polarity encounter each other and partially annihilate at an efficient rate $V_{R}$ close to the local Alfvén speed $\left(V_{A}\right)$. Besides successful laboratory reconnection experiments [22] and direct observations in the earth magnetotail and solar flares, extensive numerical work has been also carried out to understand the nature of this process both in collisionless (e.g., [23]) and collisional plasmas (e.g., [24, 25]). Different mechanisms such as plasma instabilities, anomalous resistivity, and turbulence, can lead to fast reconnection. The latter process has been found to be very efficient and probably the main driving mechanism of fast reconnection in collisional MHD flows [26]. Even weak embedded turbulence causes the wandering of the magnetic field lines which allows for many independent patches to reconnect simultaneously making the reconnection rate large and independent on the local microscopic magnetic resistivity, $V_{R} \sim v_{A}\left(l_{\text {inj }} / L\right)^{1 / 2}\left(v_{\text {turb }} / v_{A}\right)^{2}$, where $l_{\text {inj }}$ and $v_{\text {turb }}$ are the injection scale and velocity of the turbulence, respectively [26]. One should thus expect that in magnetically dominated media the release of energy will result in outflow motions that in high Reynolds number media drive turbulence that will in turn speed up the reconnection further.

The break of the magnetic field topoloy by fast reconnection invloves the release of magnetic energy explosively which explains, for instance, the bursty emission in solar flares. Relativistic particles are always observed in connection with these flares suggesting that magnetic reconnection can lead to direct particle acceleration (see for a review [17]).

In analogy to diffusive shock acceleration (DSA), in which particles confined between the upstream and downstream flows undergo a first-order Fermi acceleration, [16] (hereafter GL05) proposed a similar process where trapped particles bounce back and forth between the converging magnetic fluxes of a large scale reconnection discontinuity (or current sheet). The particles gyrorotate around a reconnected magnetic field (see Figure $2 b$ in [27], gaining energy due to collisions 
with magnetic irregularities at a rate $\Delta E / E \propto V_{R} / c$ implying a first-order Fermi process with an exponential energy growth after several round trips (GL05). This process has been extensivelly tested numerically mainly through two-dimensional particle-in-cell (PIC) simulations of collisionless plasmas [28, 29, 30, 31, 32, 33, 34, 35, 36], and more recently also through three-dimensional (3D) PIC simulations $[37,38,39]$. However, these simulations can probe acceleration only at the kinetic scales of the plasma, of a few hundreds of the inertial length $\left(\sim 100 c / \omega_{p}\right.$, where $\omega_{p}$ is the plasma frequency). To assess the first-order Fermi process in the large scales of the collisional MHD flows commonly observed in astrophyisical systems, [27, 40, 41] have also successfully tested it in 2D and 3D MHD simulations injecting thousands of test particles in the reconnection domain.

Figure 1 depicts a typical trajectory of a test particle in a large scale 3D MHD magnetic discontinuity where turbulence was embedded to make reconnection fast [40, 41]. These simulations indicate an efficient particle acceleration rate $t_{\text {acc }}^{-1} \propto E^{-\alpha}$, with $0.2<\alpha<0.6$ for a vast range of values of $c / V_{\mathrm{A}} \sim 20-1000$, and an accelerated particle power-law spectrum which in the initial times of the simulation can be fitted by $N(E) \propto E^{-1,-2}$, where $E$ is the particle kinetic energy [41].

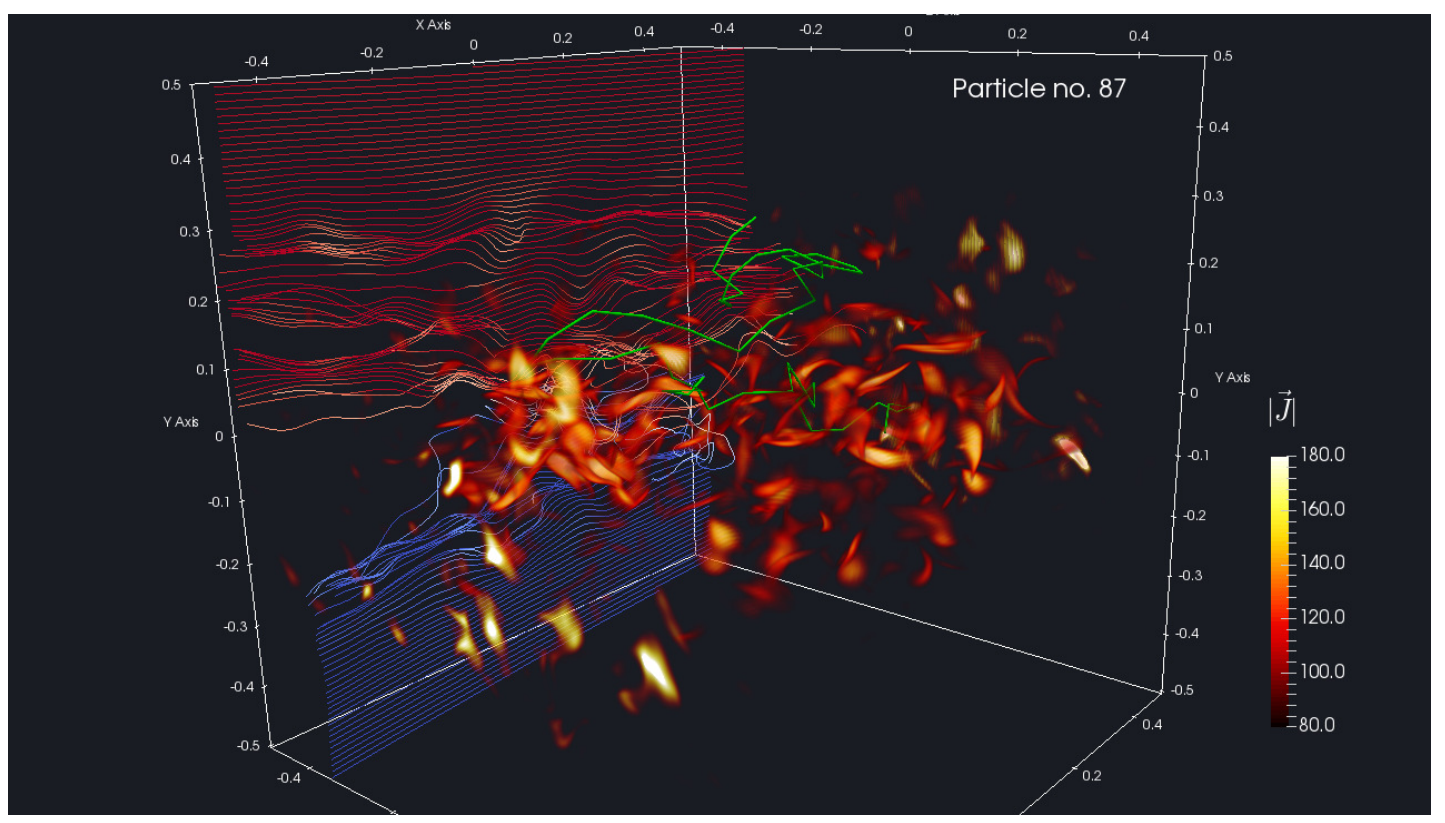

Figure 1: Map illustrating the trajectory (in green) of an injected test particle bouncing up and down in the 3D magnetic reconnection domain simulated with turbulence embedded in it to make reconnection fast. The volume depicts the current sheet region with patches of very high current density $(J)$ where the particle is scattered by the magnetic fluctuations while accelerated. The blue and red lines represent the two magnetic line fluxes of opposite polarity (extrated from [41]).

Currently, fast magnetic reconnection is regarded as an important mechanism to accelerate particles also beyond the solar system, in magnetically dominated regions of galactic and extragalactic sources like pulsars (e.g. [34, 35]), jets and the surrounds of BHs (e.g. [16, 42, 43, 44, 45, $12,19,13,30,37,38,46])$, so that the information above obtained from the numerical simulations is crucial for modelling the non-thermal emission produced out of accelerated relativistic particles 
in such sources.

In the following sections, we will discuss applications of this acceleration mechanism driven by magnetic reconnection in the framework of $\mathrm{BH}$ systems and relativistic jets.

\section{Magnetic Reconnection in the surrounds of BHs}

In earlier work (GL05) [16], we discussed a model in which particles can be accelerated through the first-order Fermi process described above, in the surrounds of a BH, in the inner coronal region, by the power extracted from fast magnetic reconnection events occurring between the field lines of the magnetosphere of the $\mathrm{BH}$ and the field lines arising from the accretion disk (see Figure 2), based on similar phenomena occurring in the solar corona. This model was first explored in the framework of microquasars and then extended to AGNs [42, 43]. These works revealed that fast reconnection could be efficient enough to produce the core radio outbursts in microquasars and AGNs. For a detailed description of the model see [12].

We should remark that recent global non-relativistic (e.g. [47, 48, 49] and general relativistic [50] MHD numerical simulations of accretion disk systems have evidenced magnetic reconnection events in the coronal region around the central source.

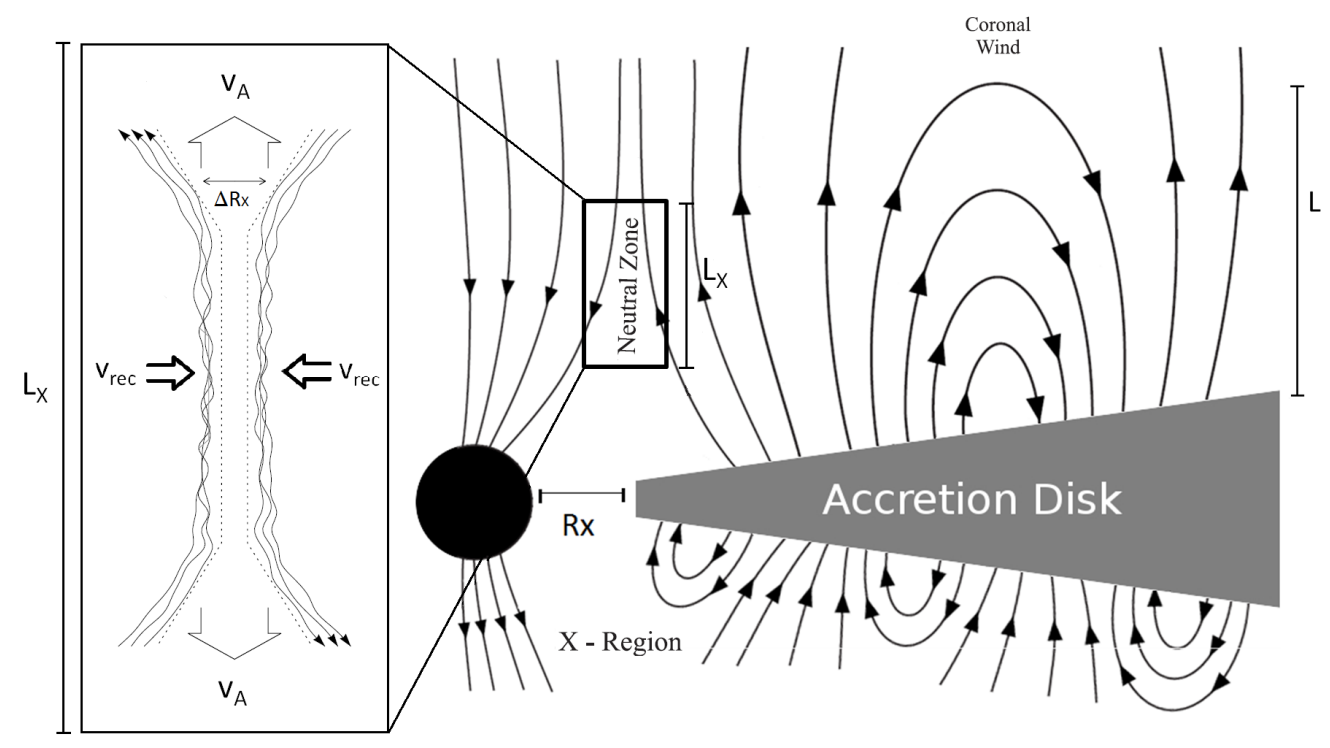

Figure 2: Idealized scheme of magnetic reconnection between the lines of the BH magnetosphere and those emerging from the accretion disk into the corona. Reconnection is made fast by the presence of embedded turbulence in the reconnection zone (as indicated in the detail) and allows for extraction of large amounts of reconnection power (eq. 1). Particle acceleration may also naturally occur in the magnetic reconnection zone by a first-order Fermi process (adapted from [12, 13]). This representation is suitable for a geometrically thin disk, but we obtain similar results when considering a magnetized ADAF-type accretion disk [18].

More recently, the model above has been revisited to explore the effects of different mechanisms of fast magnetic reconnection and accretion and to investigate the origin of the gamma-ray emission of a large sample of BH sources including AGNs, GBHs and even GRBs [12, 18]. 
The magnetic reconnection power released by fast reconnection in the magnetic discontinuity region as schemed in Figure 2 is given by [12]:

$$
W \simeq 1.66 \times 10^{35} \psi^{-0.5} r_{X}^{-0.62} l^{-0.25} l_{X} q^{-2} \dot{m}^{0.75} m \mathrm{erg} \mathrm{s}^{-1},
$$

where $m$ is the mass of the $\mathrm{BH}$ in units of solar mass, $\dot{m}$ is the accretion rate in units of the Eddington accretion, $r_{X}$ is the disk inner radius normalized by $R_{S}, l=L / R_{S}$ is the height of the corona in units of $R_{S} ; l_{X}=L_{X} / R_{S}, L_{X} \leq L$ is the extension of the magnetic reconnection zone (as shown in Figure 2), $q=\left[1-\left(3 / r_{X}\right)^{0.5}\right]^{0.25}$, and $\psi=\left[1+\left(\frac{v_{A 0}}{c}\right)^{2}\right]^{-1 / 2}$, with $v_{A 0}=B /(4 \pi \rho)^{1 / 2}, B$ being the local magnetic field, $\rho \simeq n_{c} m_{p}$ the fluid density in the corona, $n_{c}$ the coronal number density, and $m_{p}$ the proton mass. This equation has been derived considering the natural presence of turbulence in the current sheet as the driving source of fast reconnection, and taking a geometrically thin accretion disk model [12].

Figure 3 depicts this power as a function of the BH mass (gray region), considering a fiducial parametric space $\left(0.0005 \leq \dot{m} \leq 1, r_{X}=6,1 \leq l \leq 18, l_{X} \leq l\right.$, and $\left.\psi \simeq 1\right)$ [12]. The results of this study confirmed a trend predicted earlier in $[42,43]$ that there is a correlation between the calculated fast magnetic reconnection power and the $\mathrm{BH}$ mass spanning $\sim 10$ orders of magnitude. This can explain not only the observed radio, but also the gamma-ray emission from GBHs and low luminous AGNs (LLAGNs). This match is found for the emission of more than 230 sources (indicated in the diagram with red and green symbols) which include those of the so called fundamental plane of black hole activity [51]. This figure also reveals that the observed emission from blazars and GRBs does not follow the same trend as that of the LLAGNs and GBHs, indicating that the observed radio and gamma-ray emission in these cases is not produced in the core of these sources. This result is actually not a surprise, because the jet in these systems points to the line of sight and thus screens the nuclear region, so that in these sources the emission is expected to be produced by another population of accelerated particles, along the jet.

In another concomitant work [18], we explored the same mechanism, but instead of considering a standard thin, optically thick accretion disk as in the works above, we adopted a magneticallydominated advective accretion flow (M-ADAF, $[52,53]$ ) around the $\mathrm{BH}$, which may be more suitable for sub-Eddington sources. The results obtained are very similar to those of [12] depicted in Figure 3, suggesting that the details of the accretion physics are not affecting much the turbulent magnetic reconnection process which actually occurs in the corona around the $\mathrm{BH}$ and the disk.

The correlations found in Figure $3[12,18]$ have encouraged further testing of magnetic reconnection around $\mathrm{BH}$ sources. Employing the reconnection induced particle acceleration model described above and considering the relevant non-thermal loss processes of the accelerated particles (namely, Synchrotron, inverse Compton, proton-proton and proton-photon processes), we computed the spectral energy distribution (SED) of several GBHs [19] and LLAGNs [13] and found that these match very well with the observations. As an example, Figure 4 depicts the calculated SED of the radio-galaxy M87 compared with the observed fluxes from radio to gamma-rays. These results strengthen the conclusions above in favour of a core emission origin for the very high energy of these sources. The model also naturally explains the observed high variability of the emission which is produced in a very compact region. We should also remark that we have calculated the absorption of the calculated gamma-ray emission via pair creation due to interactions with photons 


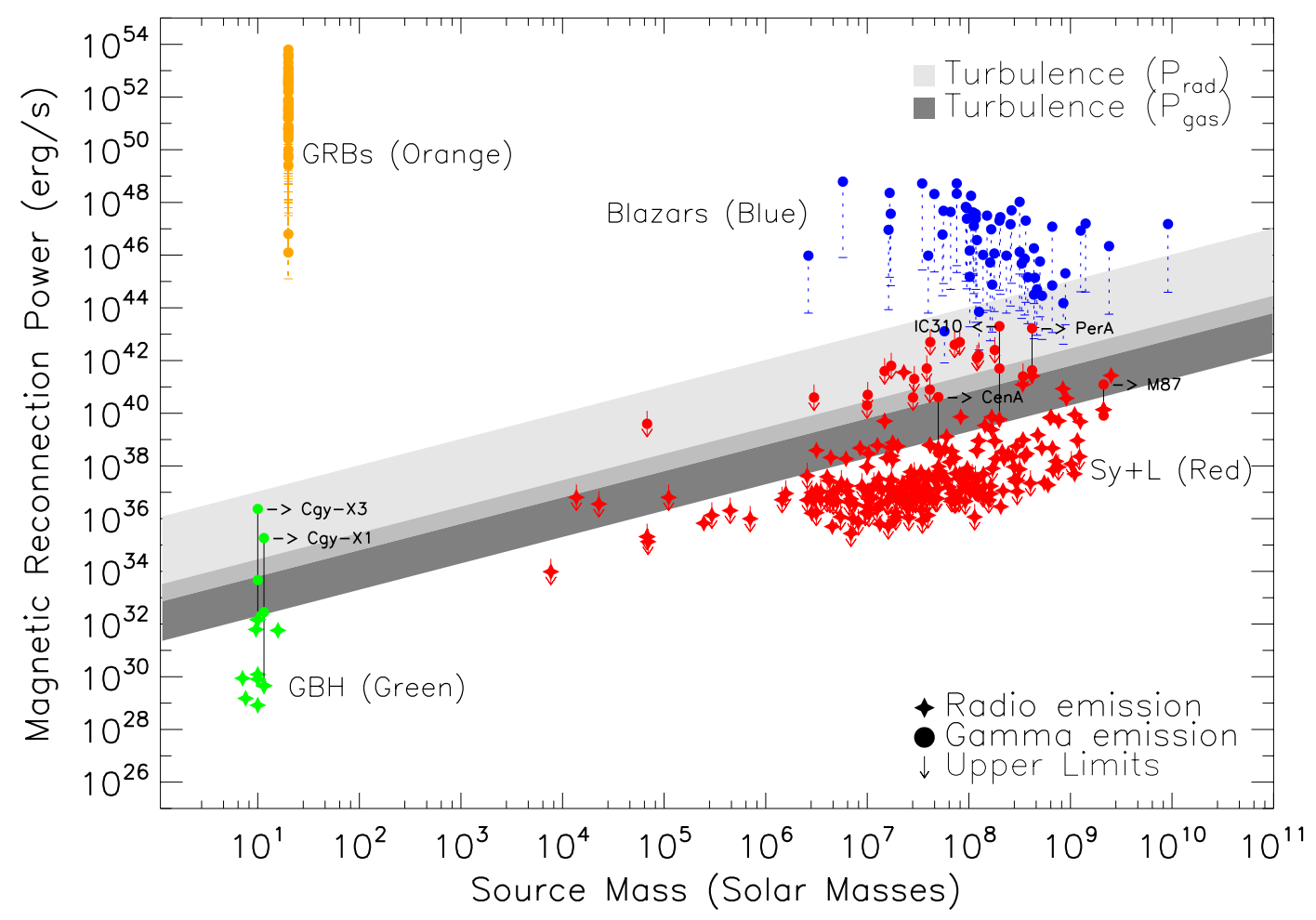

Figure 3: Calculated turbulent driven magnetic reconnection power versus BH source mass (gray region) compared to the observed emission of low luminous AGNs (LLAGNs: LINERS radio-galaxies, and Seyferts), galactic black hole binaries (GBHs), high luminous AGNs (blazars) and gamma ray burst (GRBs). The core radio emission of the GBHs and LLAGNs is represented by red and green diamonds, the gammaray emission of these two classes is represented by red and green circles, respectively. In the few cases for which there is observed gamma-ray luminosity it is plotted the maximum and minimum values linking both circles with a vertical black line that extends down to the radio emission of each of these sources. The inverted arrows associated to some sources indicate upper limits in gamma-ray emission. For blazars and GRBs, only the gamma-ray emission is depicted, represented in blue and orange circles, respectively. The vertical dashed lines correct the observed emission by Doppler boosting effects. The calculated reconnection power clearly matches the observed radio and gamma-ray emissions from LLAGNs and GBHs, but not that from blazars and GRBs. This result confirms early evidences that the emission in blazars and GRBs is produced along the jet and not in the core of the sources, but on the other hand do indicate that the gamma-ray emission of LLAGNs and GBHs can be instead produced in the core (extracted from [12]).

of the broad line region (BLR) of this source and found it to be negligible since its axis angle to the line of sight is around 30 degrees (rather than being edge-on).

The same acceleration model has been also recently applied to explain the high energy extragalactic neutrinos observed by the IceCube at TeV energies. Based on the results of [13], we have shown that protons accelerated in the core region of radio galaxies distributed between redshifts 0 and $\sim 5.2$, can produce the observed flux of neutrinos via the decay of charged pions produced by the photomeson process suffered by accelerated protons in these sources [54]. 


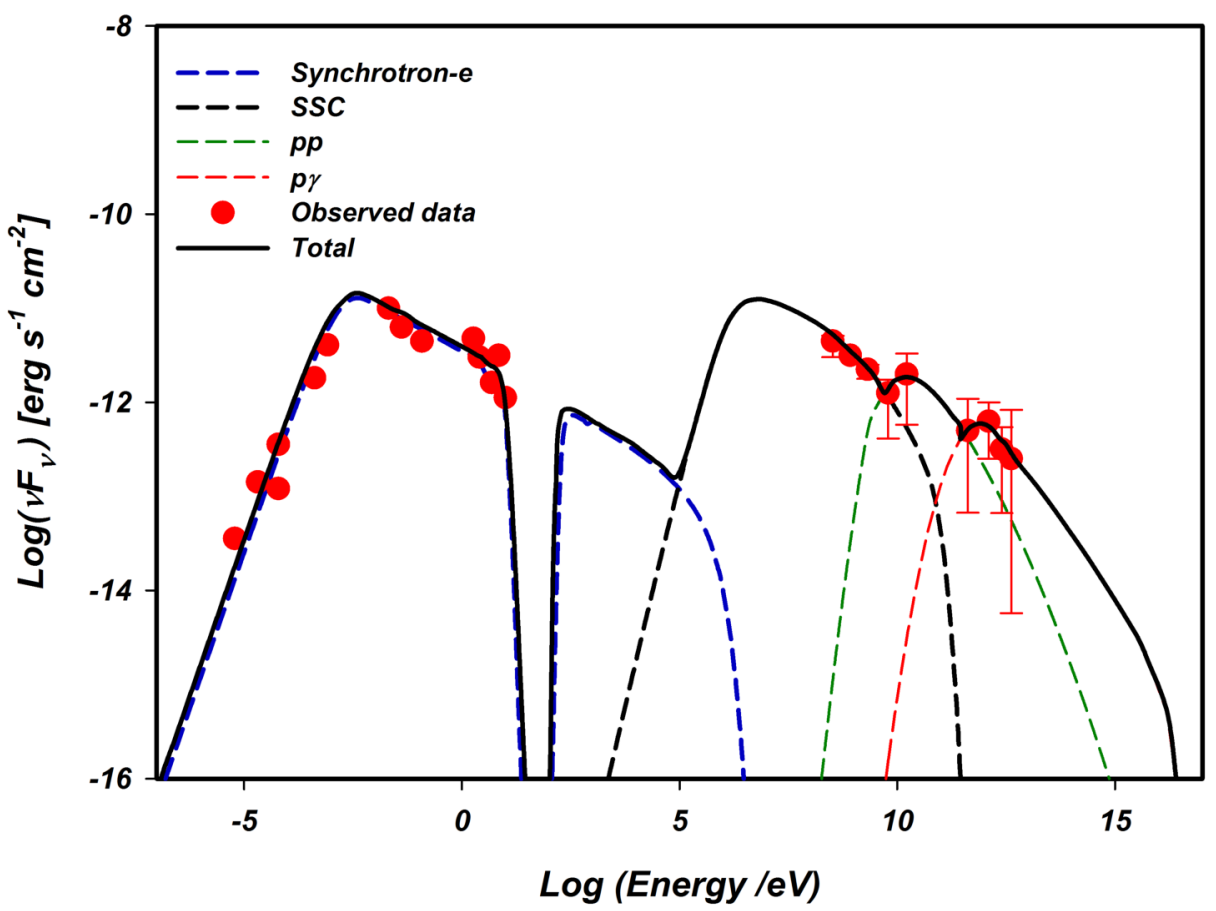

Figure 4: Calculated spectral energy distribution (SED) for the AGN M87 employing the turbulent magnetic reconnection acceleration model in the core region. The core radio data are obtained from MOJAVE VLBA at $15 \mathrm{GHz}$, [14] at 1.5, 5 and $15 \mathrm{GHz}$, IRAM at $89 \mathrm{GHz}$, SMA at $230 \mathrm{GHz}$, Spitzer at 21 and 7.2 GHz, and [15] at $3.2 \mathrm{GHz}$; optical-UV emission from HST, MeV/GeV $\gamma$-ray from Fermi-LAT; and the low-state TeV spectrum from HESS (extracted from [13]; see also references therein).

\section{Magnetic Reconnection along Relativistic Jets}

As remarked in Section 1, fast magnetic reconnection has been also lately invoked to explain the dynamical processes involved in effective conversion of magnetic into kinetic energy and on particle acceleration along relativistic jets, specially of AGNs and GRBs (for recent reviews see $[55,56]$ and references therein).

At the inner scales, around $1000 R_{S}$ from the central source or less, these jets are expected to be Poynting flux dominated flows and as such, are probably loci of strong helical fields driven by rotation at the jet launching region. Jets with strong toroidal fields are in turn susceptible to current-driven kink (CDK) instability (e.g. [57]). This excites large-scale helical motions that can strongly distort or even disrupt the beam, and induce reconnection.

To probe this process, we have performed recently 3D relativistic MHD simulations of rotating Poyinting flux dominated tower jets with initial helical fields. Considering models with a ratio between the magnetic and the rest mass energy of the flow $\sigma \simeq 1$, and different density ratios between the jet and the environment, we induced precession perturbations that quickly developed CDK modes. Figure 5 shows an example of a jet with a density larger than that of the environment. 
Decreasing density, $\Omega_{0}=2$, $\mathrm{t}=100$

(a) density

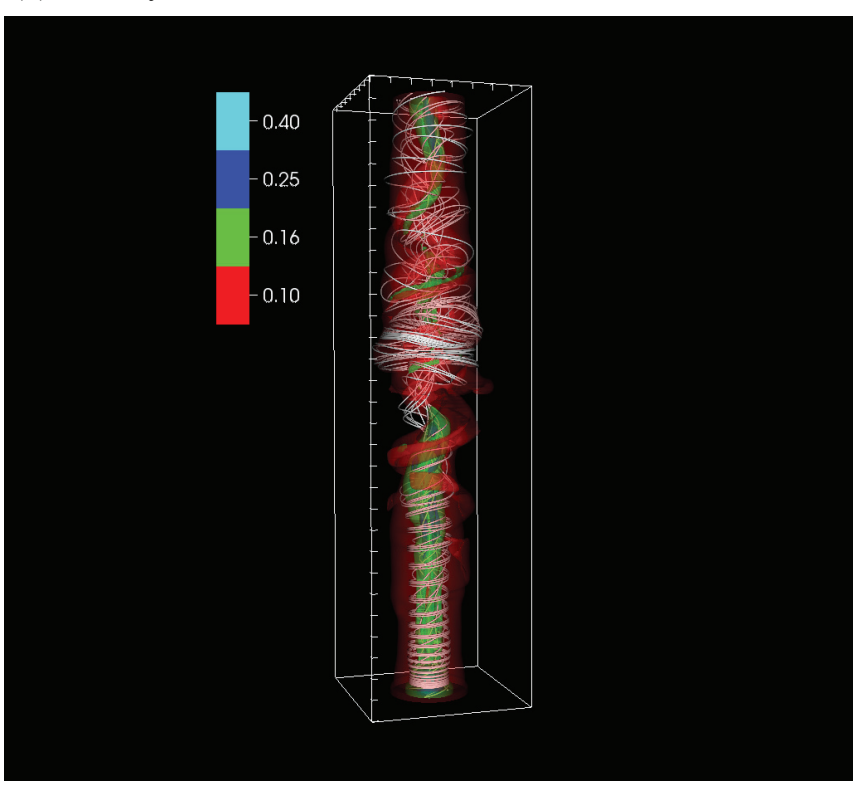

(b) curl B
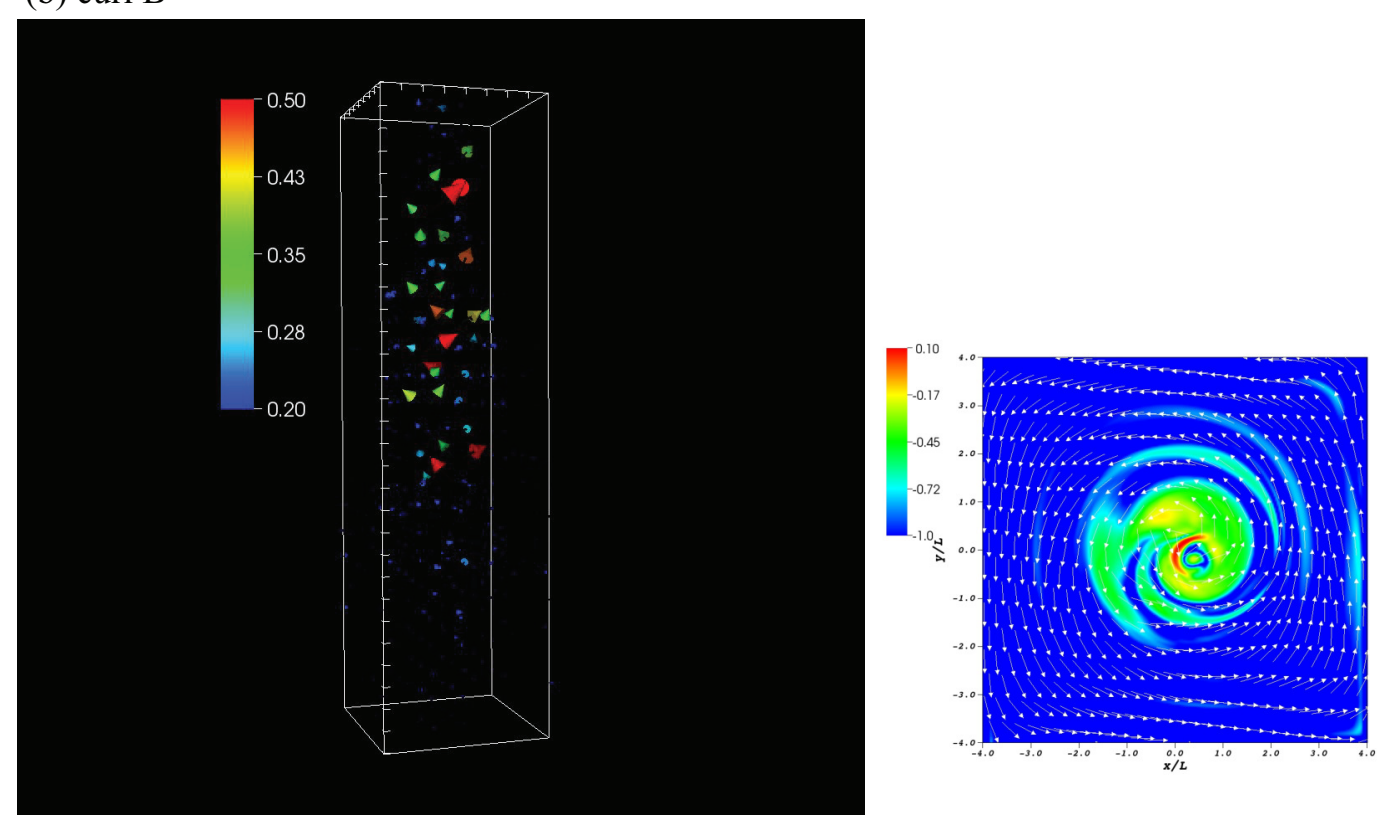

Figure 5: Current driven kink instability and fast magnetic reconnection in a relativistic MHD jet. The upper diagram depicts 3D density isosurfaces, and the bottom left diagram the locations of maximum current density which trace magnetic reconnection sites. The bottom right diagram depicts a transverse cut at $\mathrm{z}=$ 13 showing the logarithm of the current density with (white) vectors of the magnetic field superposed to it. We note clearly a large reconnection sheet near the axis (in red) which coincides with a region of minimum magnetization $(\sigma)$ in the flow due to energy dissipation (see details in [46]). 


\section{Decreasing density}
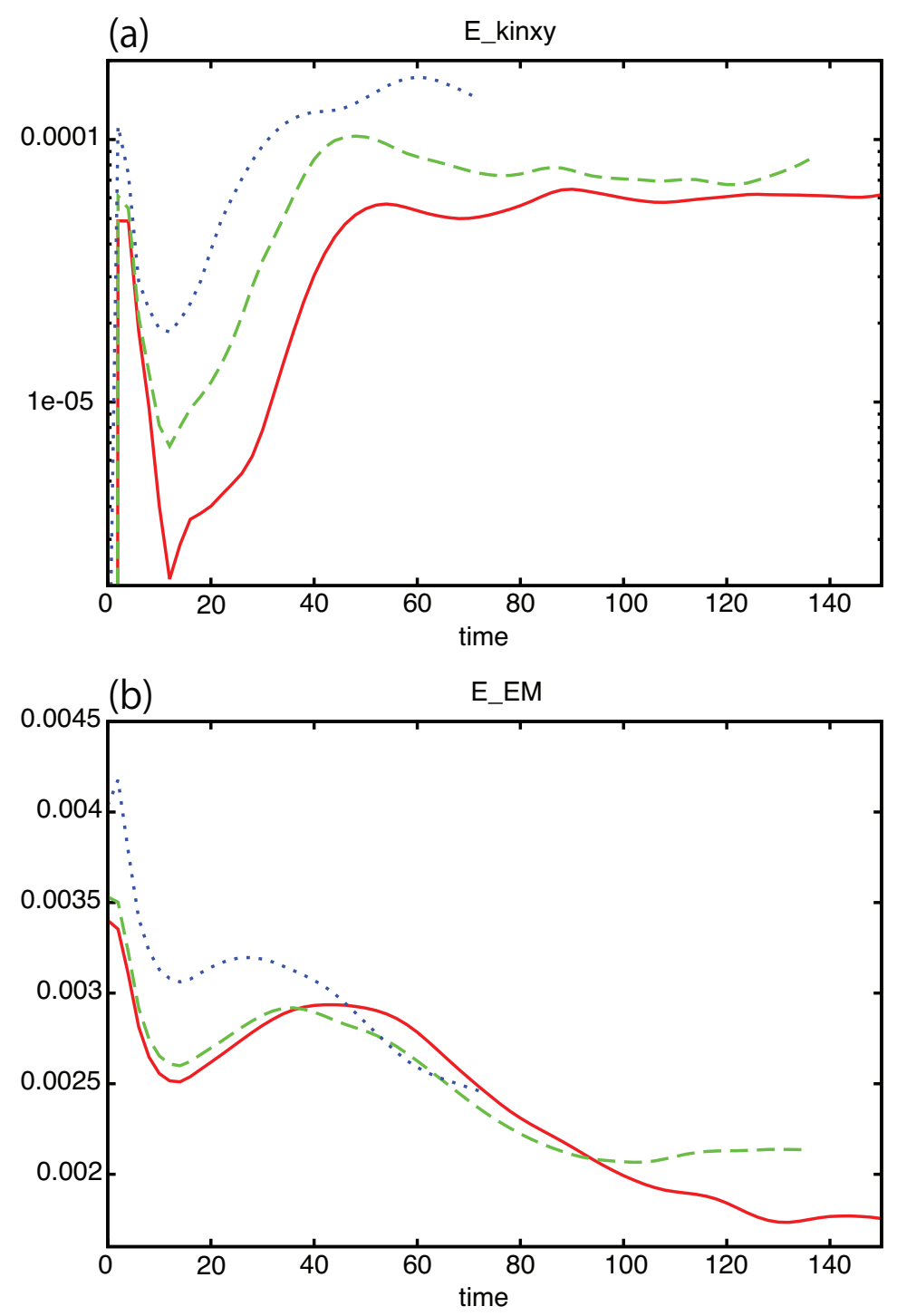

Figure 6: Volume-averaged time evolution of the kinetic (top) and magnetic (bottom) energies for the jet model presented in Figure 5 with angular velocity $\Omega=2$ (dashed green line) compared to other two models with $\Omega=1$ (solid red line), and $\Omega=4$ (blue dotted line). The depicted kinetic energy is transverse to the $\mathrm{z}$-axis, and the magnetic energy is the total integrated relativistic electromagnetic energy (extracted from [46]).

The results reveal the propagation of a helically kinked structure along the jet that causes substantial dissipation of magnetic into kinetic energy [46]. Connected to this, we identify regions of maximum current density that trace filamentary current sheets, where fast magnetic reconnection driven by the CDK instability takes place with rates $\sim 0.05 V_{A}$. This value is consistent with recent studies of fast turbulent reconnection in the relativistic regime [58] which in turn predict similar reconnection rates as those in non-relativisitc flows [26]. Figure 6 depicts the time evolution of 
the magnetic and kinetic energy of the jet flow of Figure 5 highlighting the dissipation of the first in favour of the increase of the second. We also find that the reconnection regions are directly correlated with zones of decreasing sigma in the flow.

The implications of these findings for Poynting-flux dominated jets in AGNs and GRBs are rather important since they indicate that fast magnetic reconnection can be driven by the kinkinstability turbulence and both, govern the transformation of magnetic dominated flows into kinectically dominated ones and provide an efficient way to power and accelerate particles along these relativistic jets by magnetic reconnection and explain the VHE, as predicted in earlier studies. Other concomittant similar MHD numerical studies to ours above have confirmed our findings [61, 62].

Finally, it should be mentioned that preliminary tests of in - situ particle acceleration with thousands of test particles in MHD relativistic jets (but considering small sigma values only), have revealed that magnetic reconnection acceleration can be as efficient as shock acceleration in these systems [17].

\section{Summary and Conclusions}

In this lecture, we have briefly discussed the potential role of magnetic reconnection and associated particle acceleration in the surrounds of BHs and relativistic jets, particularly stressing the importance of turbulence to induce fast reconnection.

Our main conclusions can be summarized as follows:

- First-order Fermi particle acceleration driven by fast magnetic reconnection (numerically tested $[16,27,40,41])$ can plausibly explain the observed very high energy emission of lowluminous AGNs (LLAGNs) and microquasars (or GBHs) as coming from the coronal region around the $\mathrm{BH}$ of these sources. The magnetic power released in fast reconnection events, calculated as a function of the $\mathrm{BH}$ masses (considering a fiducial parametric space depending on the size of the reconnection region and the gas accretion rate), matches very well with the observed correlation of the radio and gamma-ray luminosities of these sources with their BH masses, spanning over 10 orders of magnitude in mass and power, and tested for more than 230 sources $[12,18]$.

- Besides, the reconnection acceleration model operating in the core of these sources can also potentially reproduce the observed spectral energy distribution (SED), specially the gammaray band of these sources. This has been tested particularly for the LLAGNS and GBHs for which emission (or upper limits) at the TeV band has been detected (namely, Cen A, Per A, M87 and IC310 among the LLAGNs [13], and Cyg X1 and Cyg X3 among the GBHs [19]).

- The extragalactic very high energy neutrino flux recently observed by the IceCube has been also analysed in terms of the magnetic reconnection acceleration model above. In more speculative basis, it has been argued that this emission could be potentially explained as due to core emission from LLAGNs distributed between 0 and 5.2 redshifts [54].

- Finally, we have also discussed fast magnetic reconnection in the framework of magnetically dominated relativistic jets. 3D numerical relativistic MHD simulations of rotating tower jets with helical magnetic field subject to current driven kink instability rapidly develop fast 
turbulent reconnection regions which provide efficient magnetic energy conversion into kinetic energy, and are potential sites for efficient particle acceleretaion and strongly variable non-thermal emission, as required by current observations of GRB and AGN blazar jets [46].

A final note is in order. The origin of the VHE emission around BH sources discussed above is still debatable, specially due to the poor resolution of the current gamma-ray detectors. A core dominated origin, as suggested in this work for the observed gamma-ray emission of LLAGNs and GBHs arises as a strong possibility as long as magnetic activity is significant in the surrounds of the $\mathrm{BH}$, at the jet launching basis, and gamma-ray aborption via electron-positron pair creation is negligible, as it seems to be at the viewing angle of these sources. However, in order to ellucidate this debate, besides extensive global numerical MHD simulations of accretion disk/corona systems around BHs, we will need also substantial improvement in the observations, specially in the gamma-ray range. We hope that with the much larger resolution and sensitivity of the forthcoming gamma-ray CTA observatory [59, 60,3], and with longer times of exposure of nearby GBHs and LLAGNs, we may collect higher resolution data and more significant information on variability that may help in the determination of the true location of the emission region.

\section{Acknowledgments}

We acknowledge support from the Brazilian agencies FAPESP (2013/10559-5 grant) and CNPq (306598/2009-4 grant). The simulations presented in this lecture have made use of the computing facilities of the Laboratory of Astroinformatics (IAG/USP, NAT/Unicsul), whose purchase was made possible by FAPESP (grant 2009/54006-4).

\section{References}

[1] Blandford, R. D., \& Payne, D. G., 1982, MNRAS, 199, 883

[2] Blandford, R. D., \& Znajek, R. L. 1977, MNRAS, 179, 433

[3] Sol, H., Zech, A., Boisson, C. et al. 2013, Astroparticle Physics, 43, 215

[4] Aharonian, F. A., et al. 2006, Science, 314, 1424

[5] Abdo, A. A., et al. 2009, ApJ, 707, 55

[6] Ackermann, M., et al. 2012, ApJS, 203, 4

[7] Aleksić, J., Ansoldi, S., Antonelli, L. A., et al. 2014, Science, 346, 1080

[8] Kachelriess, M., Ostapchenko, S., \& Tomàs, R. 2010, PASA, 27, 482

[9] Tavecchio F., Ghisellini G., 2008, MNRAS, 385, L98

[10] Lenain, J. P., Boisson, C., Sol, H. \& Katarzynski, K. 2008 A\&A, 478, 111

[11] Giannios, D., Uzdensky, D. A. \& Begelman, M. C. 2010, MNRAS, 402, 1649

[12] Kadowaki, L. H. S., de Gouveia Dal Pino, E. M., \& Singh, C. B. 2015, ApJ, 802, 113

[13] Khiali, B., de Gouveia Dal Pino, E. M. \& Sol, H. 2016, A\&A (submitted); arXiv:1504.07592

[14] Biretta, J. A., Stern, C. P., \& Harris, D. E. 1991, AJ, 101, 1632 
[15] Perlman, E. S., Sparks, W. B., Radomski, J., et al. 2001, ApJ, 561, L51

[16] de Gouveia Dal Pino, E.M., \& Lazarian, A. 2005, A\& A, 441, 845 (GL05)

[17] de Gouveia Dal Pino, E. M., \& Kowal, G. 2015, in Magnetic Fields in Diffuse Media, Astrophysics and Space Science Library, Eds. A. Lazarian. E. de Gouveia Dal Pino, C. Melioli, 407, 373

[18] Singh, C. B., de Gouveia Dal Pino, E. M., \& Kadowaki, L. H. S. 2015, ApJ Letts., 799, L20

[19] Khiali, B., de Gouveia Dal Pino, E. M., \& del Valle, M. V. 2015, MNRAS, 449, 34

[20] Giannios, D., Uzdensky, D. A., \& Begelman, M. C., 2009, MNRAS, 228, 395 L29-L33

[21] Zhang, B., \& Yan, H. 2011, ApJ, 726, 90

[22] Yamada, M., Kulsrud, R., \& Ji, H. 2010, Reviews of Modern Physics, 82, 603

[23] Birn, J., \& Hesse, M. 2001, J. Geophys. Res., 106, 3737

[24] Kowal, G., Lazarian, A., Vishniac, E. T., Otmianowska-Mazur, K., 2009, ApJ, 700, 63

[25] Loureiro, N. F., Schekochihin, A. A., \& Cowley, S. C. 2007, Physics of Plasmas, 14, 100703

[26] Lazarian, A. \& Vishniac, E. T. 1999, ApJ, 517, 700

[27] Kowal, G., de Gouveia Dal Pino, E.M., \& Lazarian, A. 2011, ApJ, 735, 102

[28] Drake, J. F., Swisdak, M., Che, H., \& Shay, M. A. 2006, Nature, 443, 553

[29] Zenitani, S. \& Hoshino, M. 2001, ApJ, 562L, 63Z

[30] Zenitani, S. \& Hoshino, M. 2007, ApJ, 670, 702

[31] Zenitani, S. \& Hoshino, M. 2008, AJ, 677, 530

[32] Lyubarsky, Y. \& Liverts, M. 2008, ApJ, 682, 1436

[33] Drake, J. F., Opher, M., Swisdak, M, \& Chamoun, J. N. 2010, ApJ, 709, 963

[34] Clausen-Brown, E. \& Lyutikov, M. 2012, MNRAS, 426, 1374

[35] Cerutti, B., Uzdensky, D. A. \& Begelman, M. C. 2014, ApJ, 746, 148

[36] Li, X., Guo, F., Li, H., \& Li, G. 2015, ApJ Lett., 811, L24

[37] Sironi, L., \& Spitkovsky, A. 2014, ApJ Letts., 783, L21

[38] Guo, F., Liu, Y. H., Daughton, W. \& Li, H. 2015, ApJ, 806, 167

[39] Guo, F., Li, H., Daughton, W. \& Liu, Y. H. 2016, ApJ, Physics of Plasmas, 23, 055708

[40] Kowal, G., de Gouveia Dal Pino, E. M., \& Lazarian, A. 2012, PRL, 108, 241102

[41] del Valle, M. V., de Gouveia Dal Pino, E.M., \& Kowal, G. 2016, MNRAS (in press)

[42] de Gouveia Dal Pino, E. M., Piovezan, P. P. \& Kadowaki, L. H. S. 2010a, A\&A, 518, A5

[43] de Gouveia Dal Pino, E. M., Piovezan, P. P., Kadowaki, L. H. S., Kowal, G. \& Lazarian, A. 2010b, Highlights of Astronomy, 15, 247

[44] Giannios, D. 2010, MNRAS, 408, L46

[45] del Valle, M. V., Romero, G. E., Luque-Escamilla, P. L., Martí, J., \& Ramón Sánchez-Sutil, J. 2011, ApJ, 738, 115

[46] Singh, C. B., Mizuno, Y., \& de Gouveia Dal Pino, E. M. 2016, ApJ, 824, 48 
[47] Romanova, M. M., Ustyugova, G. V., Koldoba, A. V., \& Lovelace, R. V. E. 2011, MNRAS, 416, 416

[48] Kadowaki, L. 2011, Master Thesis, Astronomy Department, Universidade de Sao Paulo

[49] Zanni, C., \& Ferreira, J. 2013, A\&A, 550, A99

[50] Dexter, J., McKinney, J. C., Markoff, S., \& Tchekhovskoy, A. 2014, MNRAS, 440, 218

[51] Merloni, A., Heinz, S., \& di Matteo, T. 2003, MNRAS, 345, 1057

[52] Narayan R., Yi I., 1995, ApJ, 452, 710

[53] Meier, D. L., 2012, Black Hole Astrophysics: The Engine Paradigm, Springer Verlag, Berlin Heidelberg

[54] Khiali, B. \& de Gouveia Dal Pino, E. M. 2016, MNRAS, 455, 838

[55] Mizuno Y., Gomez, J.L., Nishikawa, K-I., Meli, A., Hardee, P.E., Rezzolla, L., Singh, C.B., \& de Gouveia Dal Pino, E.M., 2016, Procs. of the Conference Blazars through Sharp Multi-Wavelength Eyes, Eds. J. L. Gomez, A. P. Marscher, and S. G. Jorstad, 2016, Galaxies J. (MDPI AG) (submitted)

[56] Lazarian, A., Kowal, G., Takamoto, M., de Gouveia Dal Pino, E. M., \& Cho, J. 2016, Astrophysics and Space Science Library, 427, 409

[57] Mizuno, Y., Lyubarsky, Y., Nishikawa, K.-I., \& Hardee, P. E. 2012, ApJ, 757, 16

[58] Takamoto, M., Inoue, T., \& Lazarian, A. 2015, ApJ, 815, 16

[59] Actis, M. et al. (CTA collaboration) 2011, Exp. Astron., 32, 193

[60] Acharya, B.S. et al. (CTA collaboratioN) 2013, Astropart. Phys., 43, 3

[61] Bromberg, O., \& Tchekhovskoy, A. 2016, MNRAS, 456, 1739

[62] Striani, E., Mignone, A., Vaidya, B., Bodo, G., \& Ferrari, A. 2016, arXiv:1607.07323 